\title{
On calculating metamer sets for spectrally tunable LED illuminators
}

\author{
Graham Finlayson, ${ }^{1}$ Michal Mackiewicz, ${ }^{1, *}$ Anya Hurlbert, ${ }^{2}$ Bradley Pearce,${ }^{2}$ and Stuart Crichton ${ }^{2}$ \\ ${ }^{1}$ School of Computing Sciences, University of East Anglia, Norwich, UK \\ ${ }^{2}$ Institute of Neuroscience, Newcastle University, Newcastle, UK \\ ${ }^{*}$ Corresponding author: M.Mackiewicz@uea.ac.uk
}

Received December 13, 2013; revised April 3, 2014; accepted May 20, 2014; posted May 22, 2014 (Doc. ID 202550); published June 24, 2014

\begin{abstract}
Solid state lighting is becoming a popular light source for color vision experiments. One of the advantages of light emitting diodes (LEDs) is the possibility to shape the target light spectrum according to the experimenter's needs. In this paper, we present a method for creating metameric lights with an LED-based spectrally tunable illuminator. The equipment we use consists of six Gamma Scientific RS-5B lamps, each containing nine different LEDs and a $1 \mathrm{~m}$ integrating sphere. We provide a method for describing the (almost) entire set of illuminant metamers. It will be shown that the main difficulty in describing this set arises as the result of the intensity dependent peak-wavelength shift, which is manifested by the majority of the LEDs used by the illuminators of this type. We define the normalized metamer set describing all illuminator spectra that colorimetrically match a given chromaticity. Finally, we describe a method for choosing the smoothest or least smooth metamer from the entire set. (c) 2014 Optical Society of America

OCIS codes: (230.3670) Light-emitting diodes; (330.1715) Color, rendering and metamerism.
\end{abstract}

http://dx.doi.org/10.1364/JOSAA.31.001577

\section{INTRODUCTION}

The popularity of LED sources as commercial lighting has been steadily growing over the last decade, due in large part to their energy efficiency and longevity. Another advantage of LED sources is that they are often narrowband, and so it is easy, by combining multiple sources, to design an illuminator that has some desired spectral properties. More recently, controllable multi-LED sources have come on the market, which can generate a diverse range of different light spectra. The illuminator used in this work comprises eight narrowband LED sources arranged across the visible spectrum and two broadband yellow LEDs. However, the algorithms proposed are applicable to any similar setup.

One of the challenges of using this type of LED illuminator is that the shape of its spectral power distribution varies with the drive current. Specifically, as the current increases, the peak wavelength shifts toward longer wavelengths (sometimes by over $10 \mathrm{~nm}$ ). This shifting is illustrated in Fig. 1.

Nevertheless, given the model of the LED chromatic shifts obtained through measurements, the illuminator can be driven to meet a required target spectral shape (or a close match) or a colorimetric chromaticity match. In our earlier work, we described two algorithms capable of performing these tasks [1]].

Here we are expanding this work further and ask the question: given a target CIE $X Y Z$ (or indeed a target $x y$ chromaticity) [2], can we describe the entire set of spectra that can be created by this device? Our approach is based on the metamer sets method proposed by Finlayson and Morovic [3, 3 ] . First let us assume the ideal case that the illuminator has $N$ intensity independent LED channels. Then the illuminator spectra can be expressed as an $N$-dimensional linear model subject to certain physical inequality constraints on its weights (positive and not exceeding the channel maximum output). Finding a spectrum corresponding to a given $X Y Z$ is equivalent to solving an underdetermined system of linear equations whose solution is in the $N-3$ dimensional space. The physical inequality constraints yield a convex set in that space (a metamer set). Any spectrum in this set is a metamer, i.e., projects to form the same $X Y Z$. However, we cannot apply this approach for our device without further modifications as eight out of 10 LED channels in the RS-5B illuminator manifest intensity dependent peak-wavelength shifts.

In this paper, we model each of these eight channels using linear models at several intensity bands. The LEDs that suffer the most severely from this phenomenon are modeled using a higher number of intensity bands than those for which the problem is less significant. Next we calculate the metamer sets for every LED channel intensity band combination. Thus for a given target $X Y Z$, we calculate the metamer set for each set of intensity ranges for all our lights. The final metamer set (which is generally nonconvex) is the union of the individually calculated metamer sets. As is discussed later, the number of combinations we use, and which we determined empirically, was 1944.

Once we have calculated the metamer set of lights that project to the same $X Y Z$, it is a simple matter to find illuminants with given secondary properties. Indeed, colored objects tend to look most faithful to their appearance under natural illuminants when the metameric light chosen as a substitute has a smooth spectrum. Using optimization techniques such as quadratic programming [5] , it is a simple matter to calculate the smoothest metamer or indeed the one that is least smooth. We might also calculate the light that generates the desired target $X Y Z$ that has least total power. 


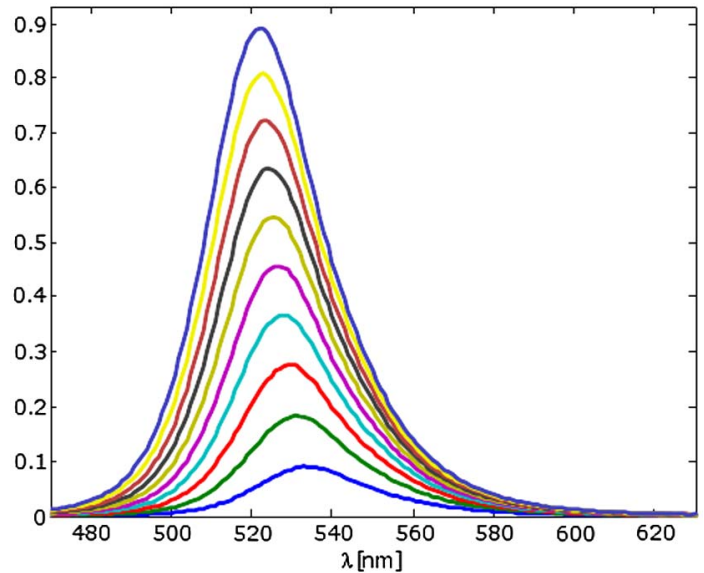

Fig. 1. Spectra of one of the LEDs for 10 intensities, ranging from the 0.1 of the maximum intensity to the maximum.

The paper is organized as follows. Section 2 gives the background information related to the calculation of metamer sets. Section 3 describes the Gamma Scientific RS-5B illuminator that we experiment with in this work. This is followed in Section 4 by the proposition of the metamer set calculation for the general case of an intensity independent set of light sources. In Section 5, we expand this method and address the problem of intensity dependent peak-wavelength shift. Next we expand the notion of the metamer set by defining the illuminator normalized metamer set that is the set of spectra producing a certain CIE $x y$ chromaticity. In Section 6 , we show how to calculate this normalized metamer set for intensity dependent illuminators. And finally, we present the algorithm for choosing the smoothest and the least smooth spectrum from the illuminator metamer set. We conclude in the final section of the paper.

\section{BACKGROUND}

Color formation can be described as a system of three linear equations that maps surface reflectances to observer responses, e.g., CIE $X Y Z$ tristimulus values:

$$
\begin{aligned}
& \chi_{1}=\int_{\omega} R(\lambda) E(\lambda) X_{1}(\lambda) \mathrm{d} \lambda, \\
& \chi_{2}=\int_{\omega} R(\lambda) E(\lambda) X_{2}(\lambda) \mathrm{d} \lambda, \\
& \chi_{3}= \int_{\omega} R(\lambda) E(\lambda) X_{3}(\lambda) \mathrm{d} \lambda ; \\
& X_{1}(\lambda)=X(\lambda), \quad X_{2}(\lambda)=Y(\lambda), \quad X_{3}(\lambda)=Z(\lambda) .
\end{aligned}
$$

Here, $R(\lambda)$ denotes a surface reflectance, $E(\lambda)$ the illuminant spectral power distribution, $X_{i}(\lambda)$ the three functions describing the observer, e.g., the CIE 1931 Standard Observer [2],$\chi_{i}$ the observer response, and $\omega$ the visible spectrum. These can be written as one equation using vector notation, where $\bar{\chi}$ is a 3-vector of observer responses:

$$
\bar{\chi}=\int_{\omega} R(\lambda) E(\lambda) \bar{X}(\lambda) \mathrm{d} \lambda .
$$

We can replace integration with the Riemann sum [ㄷ] by choosing the number of sampling points within $\omega$ :

$$
\chi_{i}=\sum_{j} x_{i j} e_{j} r_{j} \Delta \lambda,
$$

which can be written in matrix notation as in [7]:

$$
\bar{\chi}=\mathbf{X}^{T} D(\bar{e}) \bar{r} .
$$

Here the surface reflectance and the illuminant spectrum are $q \times 1$ vectors and are denoted as $\bar{r}$ and $\bar{e}$, respectively. The three observer spectral sensitivities are also $q \times 1$ vectors and are placed in the $q \times 3$ matrix $\mathbf{X}$. The matrix transpose is denoted with $T$, and $D()$ is an operator transforming a $q \times 1$ vector into a diagonal $q \times q$ matrix such that the diagonal element $(i, i)$ corresponds to the ith vector element.

There is an infinite set of reflectances that can produce the same $X Y Z$ response. These reflectances are called metamers and can be calculated by inverting the color formation equations. In [3], Finlayson and Morovic proposed a method for calculating this metamer set.

Finlayson and Morovic begin by representing a reflectance spectrum as a sum of $k<q$ basis functions. The reflectance basis is found using characteristic vector analysis [8]. Although there is not a single correct choice for $k$, the approximations that use between five to 12 basis functions are usually considered accurate enough for most applications [9-12]. The linear model representation of reflectance $\bar{r}$ can be written as

$$
\bar{r}=\mathbf{B} \bar{\sigma},
$$

where $\mathbf{B}$ is the $q \times k$ matrix of basis functions and $\bar{\sigma}$ is the $k \times 1$ vector of basis function weights.

The color formation is then simplified by adoption of Maloney's lighting matrix $\mathbf{\Lambda}^{T}=\mathbf{X}^{T} D(\bar{e}) \mathbf{B}$, which allows us to write

$$
\bar{\chi}=\Lambda^{T} \bar{\sigma}
$$

Then, finding the metamer set amounts to inverting Eq. (7), which is an underdetermined system of three linear equations with $k$ unknowns. The solution space of that system is $k-3$ dimensional and is found as the sum of two components:

$$
\bar{\sigma}=\bar{\sigma}_{p}+\bar{\sigma}_{0}
$$

where $\bar{\sigma}_{p}$ is the particular solution to

$$
\bar{\chi}=\Lambda^{T} \bar{\sigma}_{p},
$$

and $\bar{\sigma}_{0}$ is the solution to the corresponding homogeneous system and is also called the metameric black solution $[\underline{13}, 14]$ :

$$
\overline{\mathbf{0}}=\boldsymbol{\Lambda}^{T} \bar{\sigma}_{0}
$$

Many particular solutions might be chosen; for example, we could choose $\bar{\sigma}$ to be in the row space of $\Lambda$ : 


$$
\bar{\sigma}_{p}=\Lambda\left(\Lambda^{T} \Lambda\right)^{-1} \bar{\chi}
$$

Substituting $\bar{\sigma}$ defined above in Eq. (7) clearly returns the desired $X Y Z$ tristimulus value.

Finlayson and Morovic define the metamer set $\mathcal{M}(\bar{\chi})$ as

$$
\mathcal{M}(\bar{\chi})=\left\{\bar{\sigma} \mid \bar{\sigma}=\bar{\sigma}_{p}+\bar{\sigma}_{0}: \Lambda^{T} \bar{\sigma}=\bar{\chi}\right\}
$$

Solving for the metameric black component amounts to finding a $k-3$ dimensional basis $\Lambda_{0}$, which is orthogonal to $\boldsymbol{\Lambda}\left(\boldsymbol{\Lambda}_{0}^{T} \Lambda=\overline{\mathbf{0}}\right)$. There are many ways this can be done.

Let us begin by defining the orthogonal projector matrix of $\boldsymbol{\Lambda}$, which is defined as $P(\boldsymbol{\Lambda})=\boldsymbol{\Lambda}\left(\Lambda^{T} \Lambda\right)^{-1} \Lambda^{T}$ [15]. A projector has the property that if a vector $\bar{v}$ is in the space spanned by the columns of $\Lambda$ then $P(\Lambda) \bar{v}=\bar{v}$. In fact, more generally, $P(\Lambda) \bar{v}$ is the vector in the span of $\Lambda$, which is closest to $\bar{v}$. It follows then that $\bar{v}-P(\boldsymbol{\Lambda}) \bar{v}$ is that part of $\bar{v}$, which is orthogonal to the space spanned by $\Lambda$. That is, $P\left(\boldsymbol{\Lambda}_{0}\right)=\mathbf{I}-P(\boldsymbol{\Lambda})$, where $\mathbf{I}$ denotes the identity matrix.

Because we are representing reflectances with $k$ degrees of freedom, it follows that the null space projector $P\left(\Lambda_{0}\right)$ is a $k \times$ $k$ matrix that must have rank $k-3$. Thus, if we find $k-3$ vectors from the columns of $P\left(\Lambda_{0}\right)$, which are linearly independent (e.g., by casting out [16]), then we will have found the complete set of reflectances (the metameric black reflectances) that integrate to 0 :

$$
\bar{\sigma}_{0}=\Psi \bar{\alpha},
$$

where $\bar{\alpha}$ denotes any $(k-3) \times 1$ vector of weights scaling basis $\Psi$.

Assuming a general linear model of reflectance, we now have complete characterization of the set of all reflectances that integrate to form a single $X Y Z$. However, this set will contain reflectances that are not physically realizable.

In the context of Finlayson and Morovic's work, physical realizability incorporates the idea that reflectances-and later illuminants, too-are naturally bounded. They cannot reflect less than $0 \%$ of the incident light, and they cannot reflect more than $100 \%$. We write boundedness as

$$
\mathcal{P}=\{\bar{\sigma} \mid \mathbf{B} \bar{\sigma} \geq \overline{\mathbf{0}} \wedge \mathbf{B} \bar{\sigma} \leq \overline{\mathbf{1}}\},
$$

where $\overline{\mathbf{1}}$ and $\overline{\mathbf{0}}$ are $q \times 1$ vectors of ones and zeros, respectively.

Next Finlayson and Morovic define the constrained solution as the intersection [17]

$$
\mathcal{M}(\bar{\chi}) \cap \mathcal{P} .
$$

In order to impose the constraint $\mathcal{P}$ on $\mathcal{M}(\bar{\chi})$, we substitute Eqs. (8) and (13) into the reflectance positivity constraint from Eq. (14) and obtain

$$
\mathbf{B}\left(\bar{\sigma}_{p}+\Psi \bar{\alpha}\right) \geq \overline{\mathbf{0}}
$$

which can be expressed as an inequality of $\bar{\alpha}$ :

$$
\mathbf{B} \Psi \bar{\alpha} \geq-\mathbf{B} \bar{\sigma}_{p}
$$

Analogously, we form the second set of inequalities describing the upper bound on the reflectance values:

$$
\mathbf{B} \Psi \bar{\alpha} \leq \overline{\mathbf{1}}-\mathbf{B} \bar{\sigma}_{p}
$$

While these bounding constraints are easy to write down, they make calculating the metamer set much harder. Without boundedness, calculating the metamer set can be achieved using the relatively simple tools of linear algebra. Each inequality (there are $2 q$ of these) in effect forces the metamers (vectors in $k$ dimensional space) to one side of a half-space. Our $2 q$ constraints define $2 q$ half spaces, and their intersection is not a simple linear space. Rather, it will form a bounded convex region that may be delimited by many more than $2 q$ vertices [18]. However, using tools such as Qhull [19] and the duality transform of Shamos and Preparata [20], the final metamer set is readily computed.

\section{GAMMA SCIENTIFIC RS-5B ILLUMINATOR}

The illuminator system we work with has been manufactured by Gamma Scientific [21] and consists of a $1 \mathrm{~m}$ integrating sphere and six RS-5B spectrally programmable light sources (see Figs. 2 and 3). The system includes real-time optical feedback, which allows for linear brightness control. The RS-5B optical heads (each head contains all LED types) can be controlled either manually through their back panels or using the RS-5B Control Panel desktop software. This software uses a simple command protocol built on top of RS-232 ports to communicate with all six units. The RS-5B Control Panel is integrated with the SynthiColor software, which further enhances its utility by providing interactive spectral and colorimetric programming.

Each of the six optical heads located on the perimeter of the integrating sphere contains 10 LED channels. Eight of these LED channels are different narrowband sources, and the remaining two are the identical yellow phosphor broadband LED channels. The latter were incorporated into the RS-5B due to the difficulties in obtaining the narrowband LEDs operating in this part of the spectrum. The spectra of the LEDs at their maximum intensity can be seen in Fig. $\underline{4}$. Note that there are only nine spectra in this figure, as we plotted only one spectrum of the two identical yellow phosphor channels and doubled it.

The characterization of the spectral shape and luminositydrive current relationships of the LED channels, and the radiometric calibration of the LED channel outputs was performed by Gamma Scientific using standards calibrated by the National Institute of Standards and Technology (NIST). The

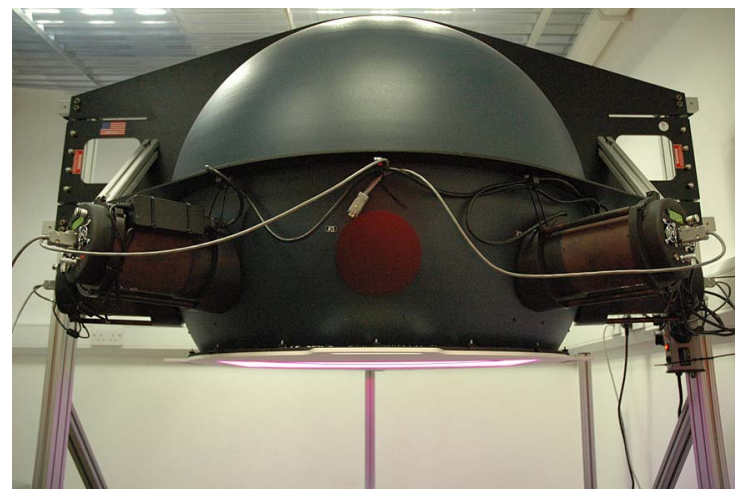

Fig. 2. RS-5B optical heads mounted onto the $1 \mathrm{~m}$ integrating sphere. 


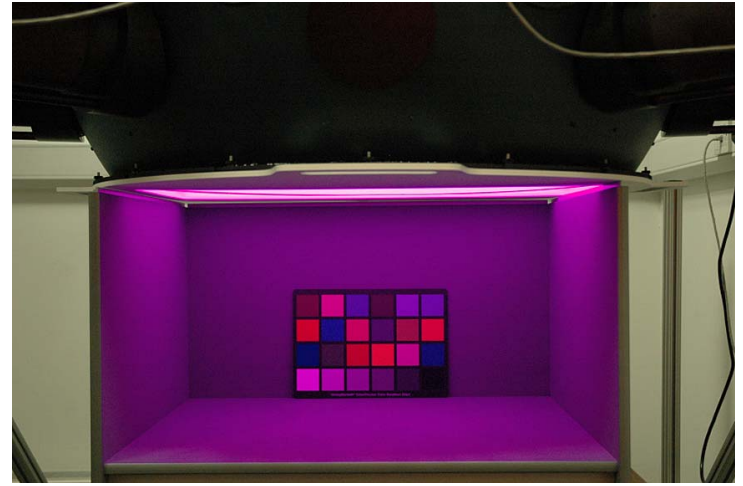

Fig. 3. RS-5B illuminating the viewing cabinet through the $34 \times 68 \mathrm{~cm}$ aperture.

luminance measured through the standard aperture for all LED channels set to max was $745 \mathrm{~cd} / \mathrm{m}^{2}$.

The LED sources exhibit an intensity (drive current) dependent peak-wavelength shift [22]. All but one (yellow phosphor) LEDs manifest this behavior. As Figs. 1 and $\underline{5}$ illustrate for one LED channel (channel 5), as the intensity increases, the peak wavelength shifts toward the shorter wavelengths. We found that, even though the spectrum shifts, the variation of all spectra may be captured by a 2D linear model, discovered using principal component analysis. Figure $\underline{6}$ illustrates the outputs of this model for channel 5 , plotting in a $2 \mathrm{D}$ space the coordinates of each spectrum shown in Fig. 1. That the resulting line is curved is indicative of a changing spectral shape along with a changing intensity.

In Fig. 7, we show the u'v' chromaticity diagram where we marked the locations of the individual LEDs at 10 different intensities by red crosses. We can see that the markers can be divided into nine clusters corresponding to the nine LED types. It is also apparent that the largest chromatic shifts occur for the green lights followed by the blue lights. The shifts in the red end of the spectrum are the smallest and, interestingly, are due to the different LED material in the opposite direction (as the intensity decreases they tend toward the shorter wavelengths). It is also clear that there is no chromatic shift for the one special LED, the yellow phosphor.

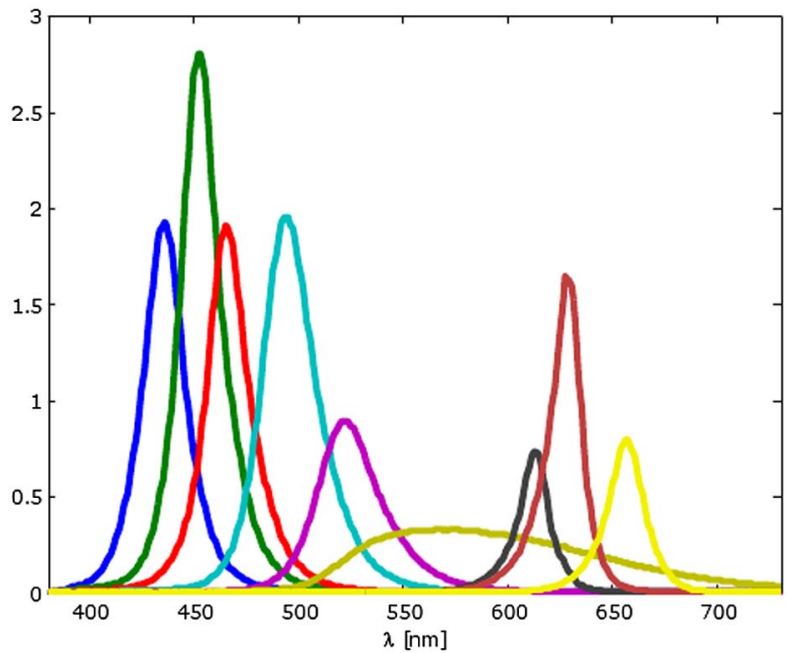

Fig. 4. Spectra of nine LEDs in RS-5B illuminator at their maximum intensity.

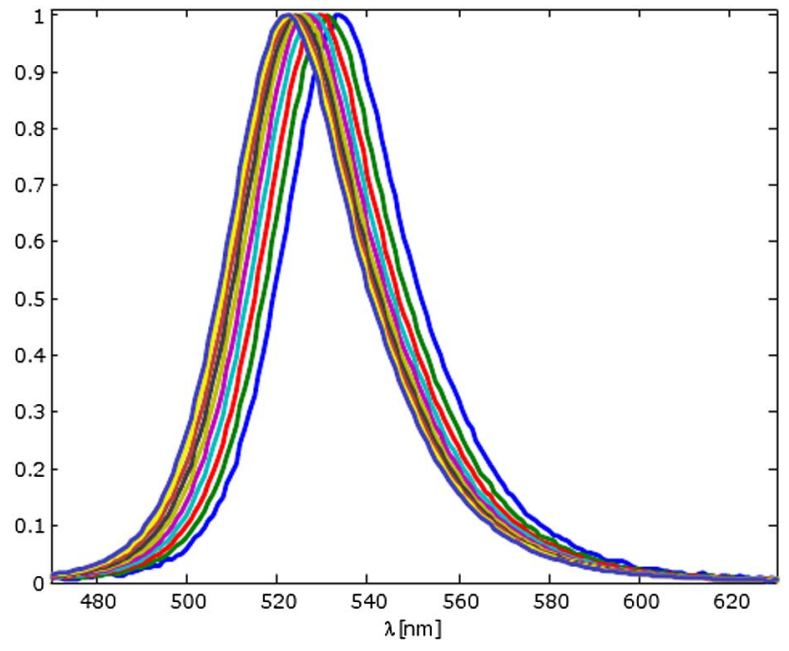

Fig. 5. Spectra from Fig. $\underline{1}$ after normalization.

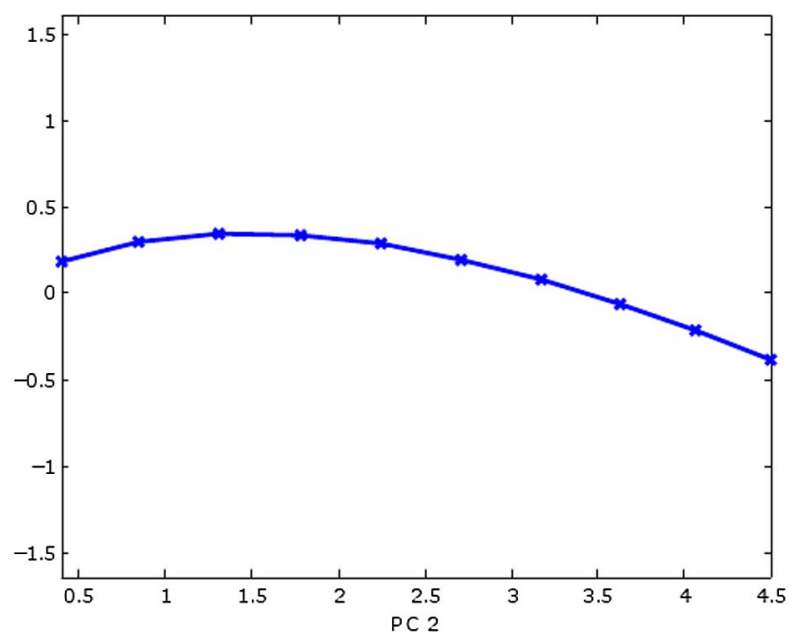

Fig. 6. First versus second principal component of the 10 LED spectra from Fig. 1 for channel 5. The 10 plotted points correspond to the 10 LED spectra of increasing intensities (from left to right).

In the next two sections, we will present the metamer set algorithms for the two types of LED illuminators: first, for those that allow for linear scaling of their channels and, second, for those that exhibit the above "spectral shifting" phenomenon.

\section{ILLUMINATOR METAMER SET: A BASIC ALGORITHM}

In this section, we discuss how to calculate the metamer set for an illuminator comprising the set of distinct sources. Here we will assume linear scaling of the light sources. First we rearrange the color formation equation:

$$
\bar{\chi}=\mathbf{X}^{T} D(\bar{r}) \bar{e} .
$$

The spectrum of illuminant $\bar{e}$ is created as the sum of $L$ linearly independent light source spectra. Analogously to the earlier reflectance spectrum decomposition, this is also a linear model representation. Unlike the reflectance linear model, however, where the choice of basis functions is arbitrary within a certain range, the number of illuminant basis 


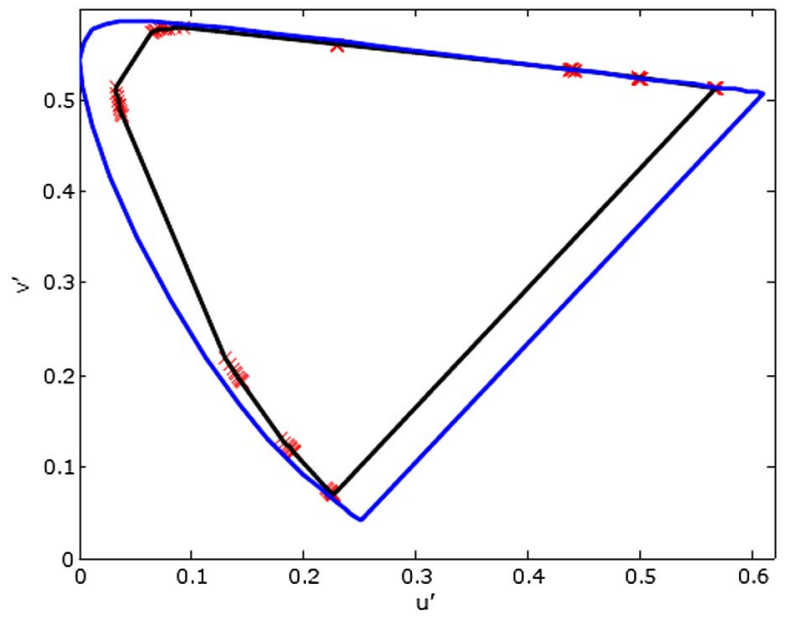

Fig. 7. Outline of the u'v' chromaticity diagram: (blue) location of LED lights for different intensities; (red x) RS-5B gamut.

functions is predetermined by the number of the physical light sources. The linear model representation of the illuminant spectrum $\bar{e}$ can be written as

$$
\bar{e}=\mathbf{C} \bar{\sigma},
$$

where $\mathbf{C}$ is the $q \times L$ matrix of basis functions (spectra of distinct physical sources) and $\bar{\sigma}$ is the $L \times 1$ vector of basis function weights.

The adoption of the $\boldsymbol{\Lambda}^{T}=\mathbf{X}^{T} D(\bar{r}) \mathbf{C}$ matrix allows us to write the color formation in the identical form to Eq. (7):

$$
\bar{\chi}=\Lambda^{T} \bar{\sigma} .
$$

Then finding the illuminator metamer set proceeds analogously to the Finlayson and Morovic method, as it amounts to inverting Eq. (21). This is an underdetermined system of three linear equations with $L$ unknowns. The solution space of that system is $L-3$ dimensional and is found as the sum of two components given by Eq. (8). Furthermore, the illuminator metamer set $\mathcal{M}(\bar{\chi})$ is given by the Eq. (12). And, finally, the particular solution $\bar{\sigma}_{p}$ and the metameric black solution $\bar{\sigma}_{0}$ can be found analogously, according to the Eqs. (11) and (13).

There is a difference in applying the physical realizability constraints. The illuminator basis functions $\mathbf{C}$ are already physical spectra at their maximum intensity. Therefore the weights of this linear model cannot be less than zero or greater than one for any basis function. We write down these constraints in the form of linear inequalities and denote the space of physically realizable solutions as

$$
\mathcal{P}_{I}=\{\bar{\sigma} \mid \bar{\sigma} \geq \overline{\mathbf{0}} \wedge \bar{\sigma} \leq \overline{\mathbf{1}}\}
$$

where $\overline{\mathbf{1}}$ and $\overline{\mathbf{0}}$ are $L \times 1$ vectors of ones and zeros, respectively.

The constrained solution is defined as the intersection

$$
\mathcal{M}(\bar{\chi}) \cap \mathcal{P}_{I}
$$

The imposition of the constraint $\mathcal{P}_{I}$ on $\mathcal{M}(\bar{\chi})$ proceeds similarly to the constraint $\mathcal{P}$ from Section $\underline{2}$. We substitute Eqs. (ㅁ) and (13) into the illuminant weight positivity constraint from Eq. (22) and obtain

$$
\bar{\sigma}_{p}+\Psi \bar{\alpha} \geq \overline{\mathbf{0}}
$$

which we can express as an inequality of $\bar{\alpha}$ :

$$
\boldsymbol{\Psi} \bar{\alpha} \geq-\bar{\sigma}_{p}
$$

Analogously, we form the second set of inequalities describing the upper bound on the weights of the illuminant linear model:

$$
\boldsymbol{\Psi} \bar{\alpha} \leq \overline{\mathbf{1}}-\bar{\sigma}_{p}
$$

The positivity and upper bound illuminant weight constraints provide two constraints per individual light source providing $2 L$ inequalities. Similarly to the reflectance metamer set solution, these inequalities constrain the solution in the space of $\bar{\alpha}$ scaling of the metameric black basis $\Psi$. The numerical solution can be obtained using the same mathematical tools that were described in Section 2 .

\section{ILLUMINATOR METAMER SET: A COMPLEX ALGORITHM}

The algorithm for calculating the illuminator metamer set described in the previous section applies to the number of illuminator setups that use either LEDs or other light sources. The only requirement is that the intensity adjustment of each component light source is linear and preserves the shape of the spectrum. As shown in Section 3, the RS-5B and in general the LED illuminators that use the $\bar{d}$ rive current for adjusting the intensity of the LEDs do not fall into this category. Therefore the chromatic shift phenomenon must be taken into account in order to calculate the metamer sets for such illuminators.

Let $l$ denote the LED channel index $l=1,2, \ldots, L$. We propose to model the LED shifting property using interpolation of the spectra measured for $K_{l}$ predefined intensities (weights) $\bar{w}_{l}$. Note, different channels can be modeled by using a different number of interpolation points; hence, index $l$ in $K_{l}$ and $\bar{w}_{l}$. Also let $q \times 1$ vector $\bar{\gamma}_{l, k}$ denote the measured spectrum of the $l$-th channel at intensity $w_{l, k}$, where $k \in\left\{1, \ldots, K_{l}\right\}$.

Then the $l$ th channel at a certain intensity $w$ can be modeled as a convex combination of the spectrum measured at some lower than $w$ intensity $w_{l, k-1}$ and higher than $w$ intensity $w_{l, k}$ :

$$
\bar{\gamma}_{l}(w)= \begin{cases}\bar{\gamma}_{l, k}(1-a)+\bar{\gamma}_{l, k-1} a, & \text { if } k \in\left\{2, \ldots, K_{l}\right\} \\ \bar{\gamma}_{l, k} a, & \text { if } k=1\end{cases}
$$

where $0 \leq a \leq 1$ and $w_{l, k-1} \leq w \leq w_{l, k}$.

In Eq. (27), the range of intensities for which we can model the channel spectrum is determined by the $\bar{w}_{l}(k-1)$ and $\bar{w}_{l}(k)$ boundary intensities. To denote this intensity band, we will use the index $k$ as $k-1$ is implicit and hence not needed. Thus, for the above equation, we can say that $w$ is in the $k$ th intensity band of the $l$ th channel. Then let $\bar{k}$ denote the $L$ vector of indices that determine the intensity bands for all $L$ channels. 
Next we define $\boldsymbol{\Gamma}(\bar{k})$ as

$$
\boldsymbol{\Gamma}(\bar{k})=\left[\begin{array}{lll}
\bar{\gamma}_{1, \bar{k}(1)-1}^{T} & - & \bar{\gamma}_{1, \bar{k}(1)}^{T} \\
\bar{\gamma}_{2, \bar{k}(2)-1}^{T} & - & \bar{\gamma}_{2, \bar{k}(2)}^{T} \\
& \vdots & \\
\bar{\gamma}_{L, \bar{k}(L)-1}^{T} & - & \bar{\gamma}_{L, \bar{k}(L)}^{T}
\end{array}\right]
$$

and $\Delta(\bar{k})$ as

$$
\boldsymbol{\Delta}(\bar{k})=\left[\begin{array}{c}
\bar{\gamma}_{1, \bar{k}(1)}^{T} \\
\bar{\gamma}_{2, \bar{k}(2)}^{T} \\
\vdots \\
\bar{\gamma}_{L, \bar{k}(L)}^{T}
\end{array}\right]
$$

Note that $\boldsymbol{\Gamma}(\bar{k})$ and $\boldsymbol{\Delta}(\bar{k})$ assume that all elements of $\bar{k}$ are greater than 1 . If any $l$-th element of $\bar{k}$ equals 1 , then its corresponding $l$-th row in matrix $\Gamma(\bar{k})$ will be $\bar{\gamma}_{l, 1}^{T}$; in matrix $\Delta(\bar{k})$, it will be $\overline{\mathbf{0}}^{T}$.

Now we can write the interpolation model of the illuminator spectrum $\bar{e}$ for a set of intensity bands defined by $\bar{k}$ as

$$
\bar{e}=\boldsymbol{\Gamma}(\bar{k}) \bar{a}+\Delta(\bar{k}) .
$$

Vector $\bar{a}$ determines the convex combination for each LED channel, and thus all its $L$ elements must not be less than 0 and greater than 1 .

We are substituting $\bar{e}$ into Eq. (19), which yields

$$
\bar{\chi}-\mathbf{X}^{T} D(\bar{r}) \Delta(\bar{k})=\mathbf{X}^{T} D(\bar{r}) \boldsymbol{\Gamma}(\bar{k}) \bar{a} .
$$

Let $\bar{\chi}^{\prime}=\bar{\chi}-\mathbf{X}^{T} D(\bar{r}) \boldsymbol{\Delta}(\bar{k})$ and $\boldsymbol{\Lambda}^{\prime T}=\mathbf{X}^{T} D(\bar{r}) \boldsymbol{\Gamma}(\bar{k})$. Then the above equation takes the familiar form of Eq. (21):

$$
\bar{\chi}^{\prime}=\Lambda^{\prime T} \bar{a} .
$$

Note that substitution of $\bar{e}$ into Eq. (19) means moving from the interpolation of the LED spectra at given intensity ranges into the interpolation of the tristimulus values of the LEDs at these intensity ranges. This also teaches that the tristimulus values of the LED spectra at those chosen intensities need to be calculated only once.

Finding the metamer set for the set of intensity bands defined by $\bar{k}$ proceeds analogously to the previous section:

$$
\mathcal{M}(\bar{\chi}, \bar{k})=\left\{\bar{a} \mid \bar{a}=\bar{a}_{p}+\bar{a}_{0}: \Lambda^{\prime T} \bar{a}=\bar{\chi}^{\prime}\right\}
$$

The physical realizability constraints, which can be considered here as intensity band constraints, are expressed as a set of inequalities on $\bar{a}$ :

$$
\mathcal{P}_{I}^{\prime}=\{\bar{a} \mid \bar{a} \geq \overline{\mathbf{0}} \wedge \bar{a} \leq \overline{\mathbf{1}}\}
$$

The constrained solution $\mathcal{C}(\bar{\chi}, \bar{k})$ is defined as the intersection

$$
\mathcal{C}(\bar{\chi}, \bar{k})=\mathcal{M}(\bar{\chi}, \bar{k}) \cap \mathcal{P}_{I}^{\prime},
$$

which can be expressed as the two sets of inequalities corresponding to Eqs. (25) and (26):

$$
\Psi^{\prime} \bar{\alpha}^{\prime} \geq-\bar{a}_{p},
$$

$$
\Psi^{\prime} \bar{\alpha}^{\prime} \leq \overline{\mathbf{1}}-\bar{a}_{p},
$$

where $L \times L-3$ matrix $\boldsymbol{\Psi}^{\prime}$ is the $L-3$ dimensional basis orthogonal to $\Lambda^{\prime}$.

The solution set expressed using $\bar{\alpha}^{\prime}$ scalings of $\Psi^{\prime}$ can be formulated in terms of $\bar{a}$ and then as intensity scalings $\bar{w}$ of individual LED channels using the equation below:

$$
w_{l}=\left\{\begin{array}{ll}
w_{l, k}\left(1-a_{l}\right)+w_{l, k-1} a_{l}, & \text { if } k \in\left\{2, \ldots, K_{l}\right\} \\
w_{l, k} a_{l}, & \text { if } k=1
\end{array} .\right.
$$

So far we showed how to calculate the metamer set for one set of $L$ intensity bands [Eq. (35)]. Clearly the final metamer set is the union of the metamer sets calculated for all intensity band permutations:

$$
\mathcal{F}(\bar{\chi})=\bigcup_{i=1}^{N} \mathcal{C}\left(\bar{\chi}, \bar{k}_{i}\right) .
$$

The number of permutations $N$ and hence the number of metamer sets that need to be calculated equals to $\Pi_{l=1}^{L} K_{l}$. Hence for an $L$ channel illuminator with each channel divided into $K$ intensity bands, one needs to calculate $K^{L}$ metamer sets.

The above calculations show the number of metamer sets that need to be calculated can be large, and this implies a significant computational overload. However, in Section 8 we will show that a relatively coarse interpolation of the intensity bands suffices, for our purposes, to achieve metamer sets with low error. Moreover, for many combinations of the intensity bands, the metamer sets are empty, and this reduces the amount of calculations even further.

\section{ILLUMINATOR NORMALIZED METAMER SET}

It is clear that for an illuminator that allows for linear channel scaling, i.e., fulfilling the assumption of the basic algorithm, the shape of the spectrum does not change when the channel weights are scaled by some factor $\beta$. Thus, as long as after multiplication by this factor the channel weights remain between 0 and 1 , the chromaticity of the illuminant remains the same. This is obviously not the case for the RS-5B illuminator discussed here. It is this observation that leads us to ask the following question: what is the set of all illuminator spectra that produce some chromaticity $x y$ ? We will call this set the illuminator normalized metamer set. Below, we will show that this set can be calculated using the same interpolation approach that we used in the complex algorithm.

Recall Eq. (19). In the current scenario, we modify this equation by the addition of the free parameter $\beta$. Thus we are looking for such spectra $\bar{e}$, which induce the color response $\beta \bar{\chi}$ for $\beta>0$ :

$$
\beta \bar{\chi}=\mathbf{X}^{T} D(\bar{r}) \bar{e} .
$$

We substitute Eq. (30) into Eq. (40):

$$
\beta \bar{\chi}-\mathbf{X}^{T} D(\bar{r}) \Delta(\bar{k})=\mathbf{X}^{T} D(\bar{r}) \boldsymbol{\Gamma}(\bar{k}) \bar{a} .
$$


We move the free parameters $\beta$ and $\bar{a}$ onto the left side of the equation:

$$
\mathbf{X}^{T} D(\bar{r}) \boldsymbol{\Gamma}(\bar{k}) \bar{a}-\beta \bar{\chi}=-\mathbf{X}^{T} D(\bar{r}) \Delta(\bar{k}) .
$$

Next we put the free parameters into a single vector:

$$
\left[\mathbf{X}^{T} D(\bar{r}) \boldsymbol{\Gamma}(\bar{k}), \quad-\bar{\chi}\right]\left[\begin{array}{l}
\bar{a} \\
\beta
\end{array}\right]=-\mathbf{X}^{T} D(\bar{r}) \mathbf{\Delta}(\bar{k}) .
$$

We will denote the first matrix in the above equation as $\boldsymbol{\Lambda}^{\prime \prime T}$, the second as $\bar{a}^{\prime \prime}$, and the right side of the equation as $\bar{\chi}^{\prime \prime}$. Then the equation takes the familiar form of Eq. (21):

$$
\boldsymbol{\Lambda}^{\prime \prime T} \bar{a}^{\prime \prime}=\bar{\chi}^{\prime \prime}
$$

Finding the normalized metamer set for the set of intensity bands defined by $\bar{k}$ proceeds analogously to the previous section:

$$
\mathcal{M}_{N}(\bar{\chi}, \bar{k})=\left\{\bar{a}^{\prime \prime} \mid \bar{a}^{\prime \prime}=\bar{a}_{p}^{\prime \prime}+\bar{a}_{0}^{\prime \prime}: \Lambda^{\prime \prime T} \bar{a}^{\prime \prime}=\bar{\chi}^{\prime \prime}\right\} .
$$

Note that the solution space of Eq. (44) is $L-2$ dimensional as opposed to the earlier $L-3$ dimensional solution of Eq. (32).

The physical realizability constraints are the same as in Eq. (34).

The constrained solution $\mathcal{C}_{N}(\bar{\chi}, \bar{k})$ is defined as the intersection of $\mathcal{M}_{N}(\bar{\chi}, \bar{k})$ and $\mathcal{P}_{I}^{\prime}$. Again, it can be expressed as the two sets of inequalities. These inequalities enforce the constraints on the first $L$ elements of the solution $L+1$ element vector $\bar{a}^{\prime \prime}$, i.e., $L$-vector $\bar{a}$ :

$$
\begin{gathered}
\boldsymbol{\Psi}^{\prime \prime} \bar{\alpha}^{\prime \prime} \geq-\bar{a}_{p}, \\
\boldsymbol{\Psi}^{\prime \prime} \bar{\alpha}^{\prime \prime} \leq \overline{\mathbf{1}}-\bar{a}_{p} .
\end{gathered}
$$

Matrix $\Psi^{\prime \prime}$ is formed from the first $L$ rows of the $L+1 \times$ $L-2$ matrix being the $L-2$ dimensional basis orthogonal to $\Lambda^{\prime \prime}$ and $\bar{\alpha}_{p}$ contains first $L$ elements of $L+1-$ vector $\bar{a}_{p}^{\prime \prime}$.

The solution set expressed using $\bar{\alpha}^{\prime \prime}$ scalings of $\Psi^{\prime \prime}$ can be formulated in terms of $\bar{a}$ and then as intensity scalings $\bar{w}$ of individual LED channels as in Eq. (27). The final normalized metamer set is the union of the normalized metamer sets calculated for all intensity band permutations:

$$
\mathcal{F}_{N}(\bar{\chi})=\bigcup_{i=1}^{N} \mathcal{C}_{N}\left(\bar{\chi}, \bar{k}_{i}\right)
$$

\section{METAMER SET SMOOTHEST SPECTRUM}

It is often required to select an element of the metamer set that meets certain characteristics of which spectrum smoothness is an important example. Here we propose an algorithm for selecting the smoothest or the least smooth member of the metamer set. The algorithm is based on the numerical method of estimating the smoothest reflectance proposed by $\mathrm{Li}$ and Luo [23].

The measure of smoothness of the spectrum $E(\lambda)$ can be expressed as

$$
\int_{\omega}\left(\frac{d E}{d \lambda}\right)^{2} \mathrm{~d} \lambda \approx\|\mathbf{G} \bar{e}\|^{2} / \Delta \lambda
$$

where $\mathbf{G}$ is an $q-1 \times q$ matrix and is given by

$$
\mathbf{G}=\left[\begin{array}{ccccc}
-1 & 1 & 0 & \cdots & 0 \\
0 & -1 & 1 & & \vdots \\
\vdots & & \ddots & \ddots & 0 \\
0 & \cdots & 0 & -1 & 1
\end{array}\right]
$$

and $\bar{e}$ is a vector sampling spectral function $E(\lambda)$ every $\Delta \lambda$.

Since $\Delta \lambda$ is a constant, it can be removed from the optimization. Then finding the smoothest $\bar{e}$ amounts to performing the following minimization:

$$
\min _{\bar{e}}\|\mathbf{G} \bar{e}\|^{2}
$$

Let us place the $p$ spectra at the vertices of the metamer set convex hull in the columns of matrix $\mathbf{M}$. We know that any convex combination of the metamer set vertex spectra produces an element of the metamer set. Then, in the above minimization, we can substitute $\bar{e}$ for the matrix M column convex combination $\mathbf{M} \bar{a}$ and rewrite the optimization as

$$
\begin{array}{cc}
\underset{\bar{a}}{\operatorname{minimize}} & \|\mathbf{G M} \bar{a}\|^{2} \\
\text { subject to } & \overline{\mathbf{0}} \leq \bar{a} \leq \overline{\mathbf{1}} \wedge \sum_{i=1}^{p} a_{i}=1 .
\end{array}
$$

This type of optimization problem is called quadratic programming and can be solved using standard methods [24].

For the metamer set obtained from the basic algorithm, selecting the smoothest spectrum amounts to applying this optimization directly. However, for the metamer set defined as the union of the metamer subsets obtained with the complex algorithm, first we need to apply the optimization for each of the metamer subsets obtaining the smoothest metamer candidates and finally choose the smoothest spectrum from among these candidates.

\section{RESULTS}

In previous sections, we developed the theory to solve for the illuminator metamer set. Here we provide the reader with some examples of this theory. The illuminator for which we will create the metamer sets will be the aforementioned Gamma Scientific RS-5B.

Initially we calculate the illuminator metamer sets with the basic algorithm for two illuminants: D65 and A. The illuminator has nine distinct channels, which are measured at their maximum intensity (see also Fig. 4) and placed in matrix C. Given the CIE $X Y Z$ spectral sensitivities $\mathbf{X}$, a uniform perfect white reflectence $\bar{r}$, and the above defined $\mathbf{C}$, we have defined the lighting matrix $\Lambda$. Following the steps of the basic algorithm to solve for the metamer set, we first find the particular solution $\bar{\sigma}_{p}$ and metameric black basis $\Psi$. The former is plotted in Fig. 8 for the D65 and A metamers. The latter are the same for both illuminants and are plotted in Fig. 9. There are six metameric black basis vectors as $L=9$, and hence the solution space is $L-3=6$ dimensional. Note that both particular solutions (particularly visible for illuminant A) and the metameric black basis functions have negative weights 


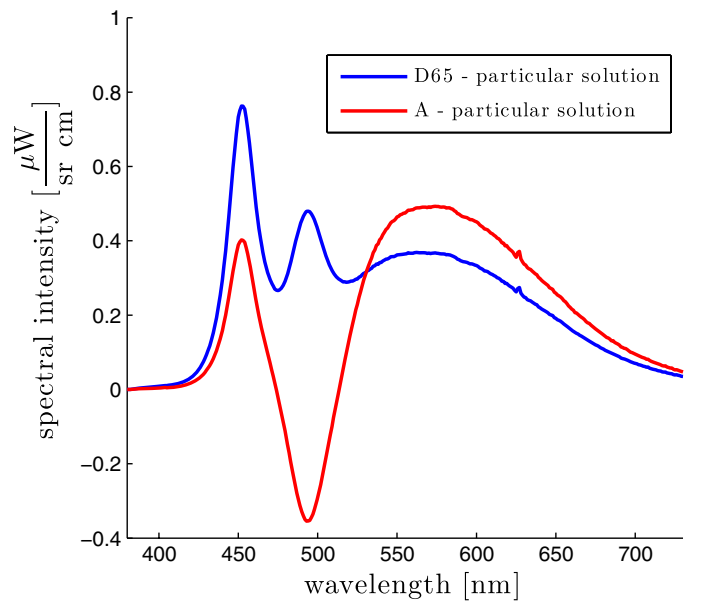

Fig. 8. Illuminant A and D65 metamer particular solutions (at $\left.246 \mathrm{~cd} / \mathrm{m}^{2}\right)$.

(or above 1) for some LED channels and hence are not physically realisable.

Then the metamer set $\mathcal{M}(\bar{\chi})$ contains elements, which are the sum of the particular solution $\sigma_{p}$ and a linear combination of the metameric black basis $\Psi \bar{\alpha}$, such that $\bar{\alpha}$ is constrained to produce a physically realizable illuminant. In Fig. 10 we plot those spectra metameric to the illuminant A (at $2 \overline{46} \mathrm{~cd} / \mathrm{m}^{2}$ ), which correspond to the vertices of the metamer set convex hull. Analogical plots for illuminant D65 can be seen in Fig. 11 .

Earlier, we noted that a basic algorithm based on a simple linear combination of the LED channels does not provide an accurate model of the RS-5B illuminator output. It is then appropriate to quantify the error of this algorithm. The maximum output luminance of the system occurs when all channels are set to maximum and equal $745 \mathrm{~cd} / \mathrm{m}^{2}$. Obviously, in this situation, i.e., at $745 \mathrm{~cd} / \mathrm{m}^{2}$, the basic model is errorless and produces only one illuminant chromaticity. In order to be able to produce a range of chromatic lights as well as allow for sizeable metamer sets, the luminance requirements need to be lowered. In Figs. 10 and 11, we illustrate the two metamer sets for illuminants $\overline{\mathrm{A}}$ and $\overline{\mathrm{D} 65}$ at $246 \mathrm{~cd} / \mathrm{m}^{2}$. We calculated the colorimetric errors for all the spectra at vertices of these two metamer sets as well as for the corresponding two metamer sets at 123 and $492 \mathrm{~cd} / \mathrm{m}^{2}$. The errors were calculated

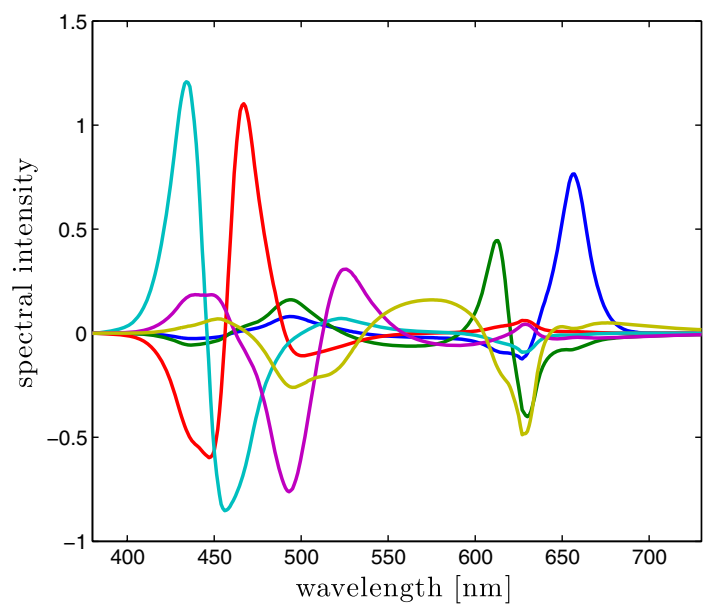

Fig. 9. Metameric black (orthogonal to $\boldsymbol{\Lambda}$ ) basis spectra.

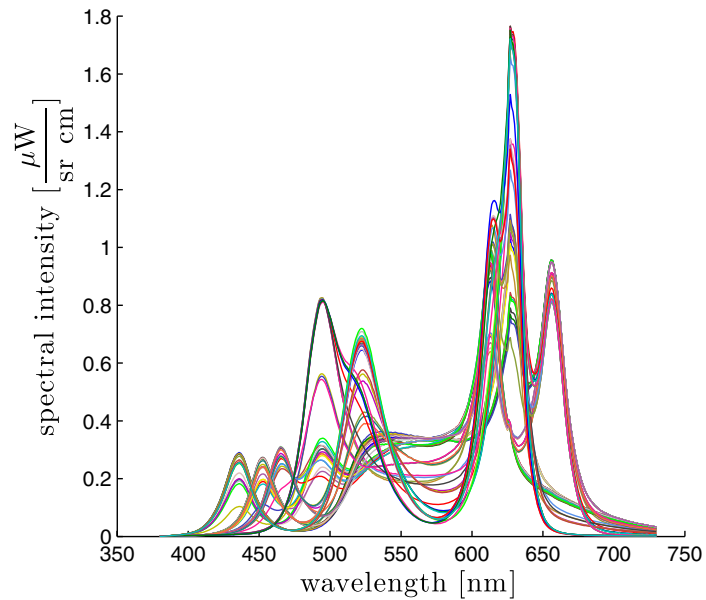

Fig. 10. Illuminant A metamers (at $246 \mathrm{~cd} / \mathrm{m}^{2}$ ).

using the full interpolation model of all LED channels. This model utilizes LED spectra measured at 11 different intensities: 0.1 to 1 with a 0.1 step and an additional measurement at 0.01 of maximum intensity. In Fig. 12, we plot the locations of the D65 and A illuminants in the CIE chromaticity diagram and the extent of the errors at the vertices of the four out of six aforementioned metamer sets. The error convex hulls of the two metamer sets at $492 \mathrm{~cd} / \mathrm{m}^{2}$ are not shown, as they are so small that they would not be visible at this figure. It is clear that the errors are significant and increase as the luminance of the metamer sets decrease. This has been expected as the basic model is based on the measurements of the channels at maximum intensity, and the lower the intensity of the channels the more significant the peak wavelength shift induced colorimetric errors.

These errors are also expressed as mean $\Delta E$ errors in the CIELAB color space [25] and are shown in Table 1. Here, apart from the mean errors at vertices of the metamer sets, we also show the mean errors at 50 random convex combinations of these vertices. Accordingly with the earlier graphic illustration, the errors become significant as the luminance of the metamer sets decrease.

The same table also contains the corresponding errors for the complex algorithm, which were obtained as follows. First we need to establish the interpolation bands for different LED

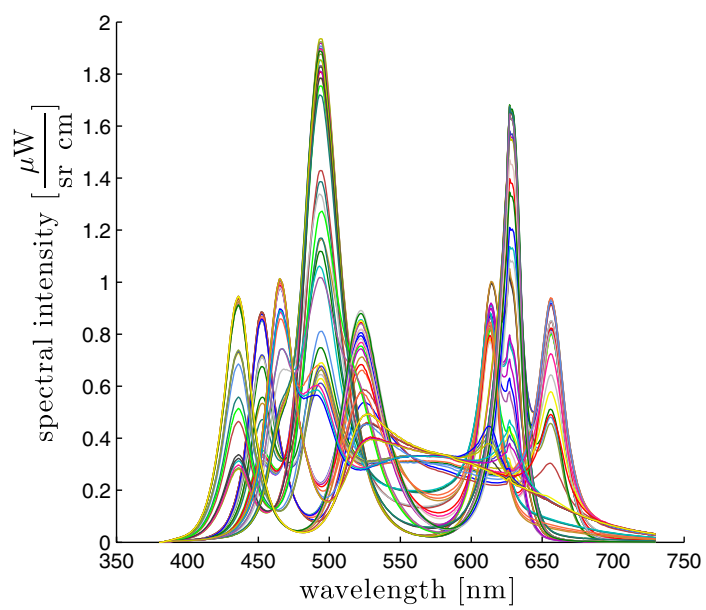

Fig. 11. Illuminant D65 metamers (at $246 \mathrm{~cd} / \mathrm{m}^{2}$ ). 


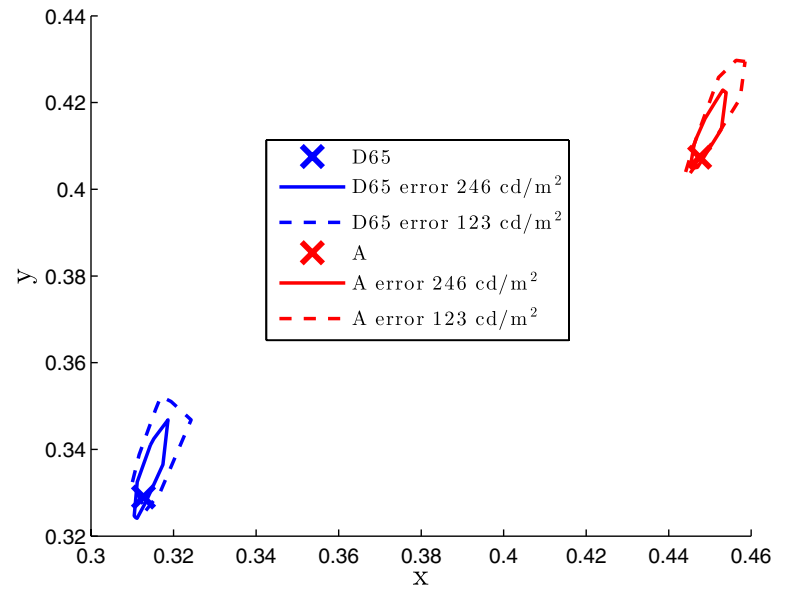

Fig. 12. Illuminant D65 and A CIE 1931 chromaticities, x; solid and dashed lines represent the convex hulls of metamers at vertices of the metamer sets for 246 and $123 \mathrm{~cd} / \mathrm{m}^{2}$, respectively.

channels. In Section 5, we mentioned that these can be different for different channels. We cannot use as many bands as we used for verification of the results since having 11 interpolation bands for each of the nine channels would result in $11^{9}$ metamer sets, which would take too long to calculate and later process. Therefore it is important to lower this number while attempting to maintain a high degree of accuracy. In our case, we decided to divide the first five LED channels (starting from the shortest wavelength, see Fig. 4) into three interpolation bands split at intensities: $0.2,0.5$, and 1 . The sixth channel is the broadband yellow phosphor, which does not exhibit the intensity dependent peak-wavelength shift and hence is represented as in the basic algorithm, i.e., only at maximum intensity. The remaining three channels at the long wavelength end of the spectrum are modeled using two interpolation bands, each split at intensities 0.3 and 1 . The reason for using a smaller number of interpolation bands here is the different technology of the LEDs, which, as shown in Fig. $\underline{7}$, results in significantly smaller peak wavelength shifts for these three channels. Hence the number of interpolation band permutations is $N=3^{5} 2^{3}=1,944$. Table 1 teaches that a relatively coarse interpolation of the channel intensities results in the metamer sets demonstrating the level of accuracy that should be adequate for most applications.

Figure 13 contains the spectra at the vertices of the metamer set calculated for one of the 1944 interpolation bands. All spectra are contained between this particular band's upper and lower envelopes. The envelopes are the sums of measured spectra at the lower end of all bands (lower envelope) and the

Table 1. Mean $\Delta E$ Errors Calculated for All Vertices of the Metamer Sets (v) and for Random Convex Combinations of These Vertices (cc) for Basic and Complex Algorithms at Three Luminance Levels

\begin{tabular}{|c|c|c|c|c|c|c|c|}
\hline \multirow[b]{2}{*}{ Illuminant } & \multirow[b]{2}{*}{ Model } & \multicolumn{2}{|c|}{$123 \mathrm{~cd} / \mathrm{m}^{2}$} & \multicolumn{2}{|c|}{$246 \mathrm{~cd} / \mathrm{m}^{2}$} & \multicolumn{2}{|c|}{$492 \mathrm{~cd} / \mathrm{m}$} \\
\hline & & $\mathrm{v}$ & $c c$ & $\mathrm{v}$ & $\mathrm{cc}$ & $\mathrm{v}$ & $\mathrm{cc}$ \\
\hline A & Basic & 7.8 & 10.1 & 5.0 & 8.0 & 1.7 & 1.7 \\
\hline D65 & Basic & 8.3 & 12.2 & 3.8 & 8.5 & 1.7 & 4.6 \\
\hline A & Complex & 0.6 & 1.5 & 0.4 & 0.8 & 0.1 & 0.2 \\
\hline D65 & Complex & 0.5 & 0.8 & 0.5 & 0.7 & 0.4 & 0.6 \\
\hline
\end{tabular}

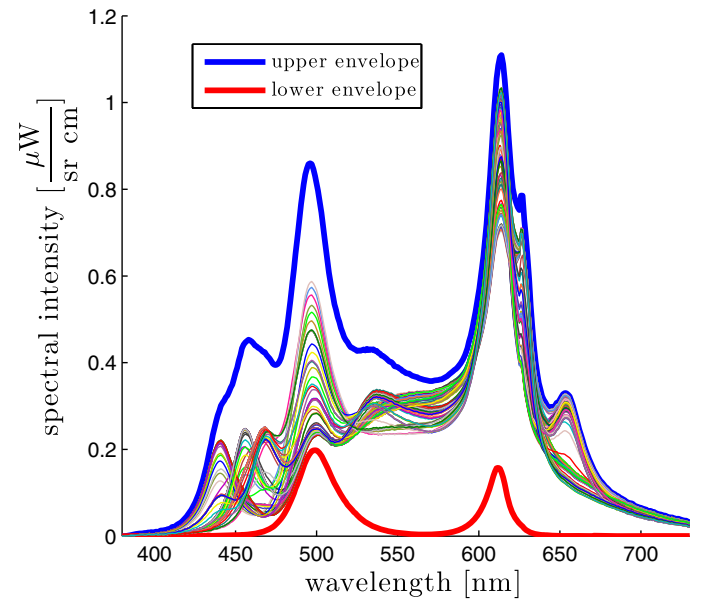

Fig. 13. Illuminant A metamers (at $246 \mathrm{~cd} / \mathrm{m}^{2}$ ) calculated for one of the interpolation bands. The thick red and blue lines denote the lower and upper envelopes of this particular interpolation band.

upper end of the bands (upper envelope). The analogous, although for a different set of interpolation bands, result for the D65 metamer set can be seen in Fig. 14. Both metamer sets were calculated for the same luminance, $246 \mathrm{~cd} / \mathrm{m}^{2}$.

As to the error of the complex algorithm (see two bottom rows in Table 1 ), we can see a significant improvement over the basic algorithm. The errors do not depend on the luminance of the metamer set, and, in the majority of cases, the mean CIELAB errors are below $1 \Delta E$.

An alternative look at the errors induced by the basic and complex algorithms would be to look at the metamer mismatch volumes [26,27] resulting from these two algorithms. In our scenario, metamer mismatch volumes arise when the perfect white reflectance illuminated by the lights from the metamer set is replaced by any nonuniform reflectance. Then the illuminant spectra coming from a metamer set cease to induce a unique sensor response. Figure 15 contains the illustration of the $2 \mathrm{D}$ metamer mismatch volumes for the basic and complex algorithms after the metamer sets were projected to reflectance no. 4 (green patch) from the Macbeth ColorChecker Chart. We can see that the erroneous metamer sets obtained using the basic algorithm result in a significantly different metamer mismatch volume than the union of the

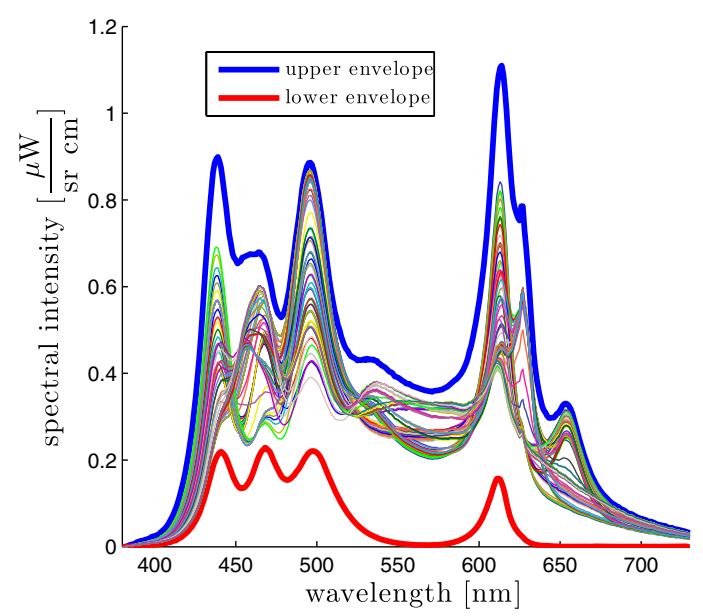

Fig. 14. Illuminant D65 metamers (at $246 \mathrm{~cd} / \mathrm{m}^{2}$ ) calculated for one of the interpolation bands. 


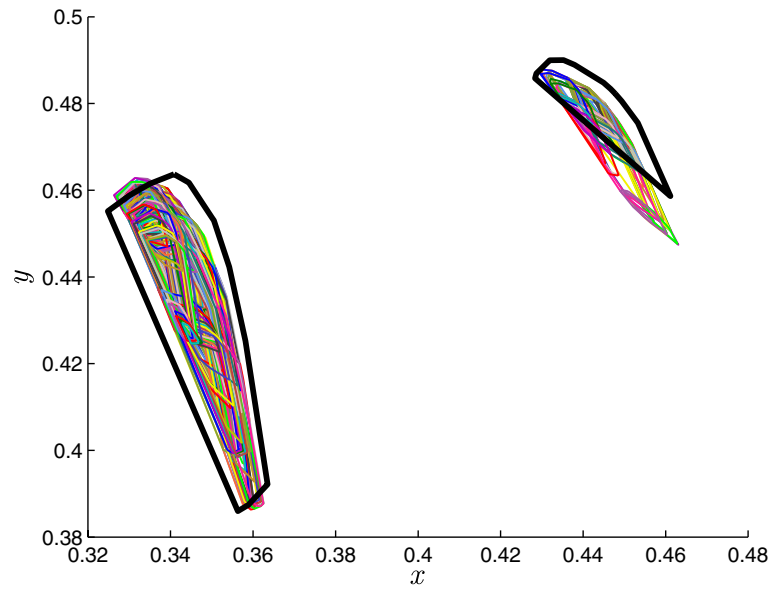

Fig. 15. Illuminant A and D65 (both at $246 \mathrm{~cd} / \mathrm{m}^{2}$ ) 2D metamer mismatch volumes for the fourth (dark green) patch from the Macbeth CC. Thick black lines denote the two volumes calculated with the basic model, and the color lines denote the subvolumes calculated with the complex model.

relatively precise metamer sets calculated for all interpolation band permutations using the complex algorithm.

In Fig. 16, we can see the spectra at the vertices of the illuminant $\mathrm{A}$ normalized metamer set calculated for one of the interpolation bands with the algorithm presented in Section 6 . The same result for the illuminant D65 can be see in Fig. 17 . There is a clear difference between these two sets of spectra and those in Figs. 13 and 14. We can see that, as expected, the spectra in the normalized sets vary in terms of intensity. For obvious reasons, the normalized metamer sets are much larger than the metamer sets calculated for some fixed luminance. In these sets of experiments, for the illuminant A the metamer sets were nonempty in 221 (normalized) and 121 (fixed at $246 \mathrm{~cd} / \mathrm{m}^{2}$ ) of 1944 interpolation bands. The same figures for D65 were 1143 and 664 .

Finally, we calculated the smoothest and the least-smooth spectra metameric to the illuminants D65 and A at $246 \mathrm{~cd} / \mathrm{m}^{2}$ (see Fig. 18). Each spectrum in this figure was obtained by performing a sequence of optimizations as described in Section 7 for each of the subsets of the metamer set resulting

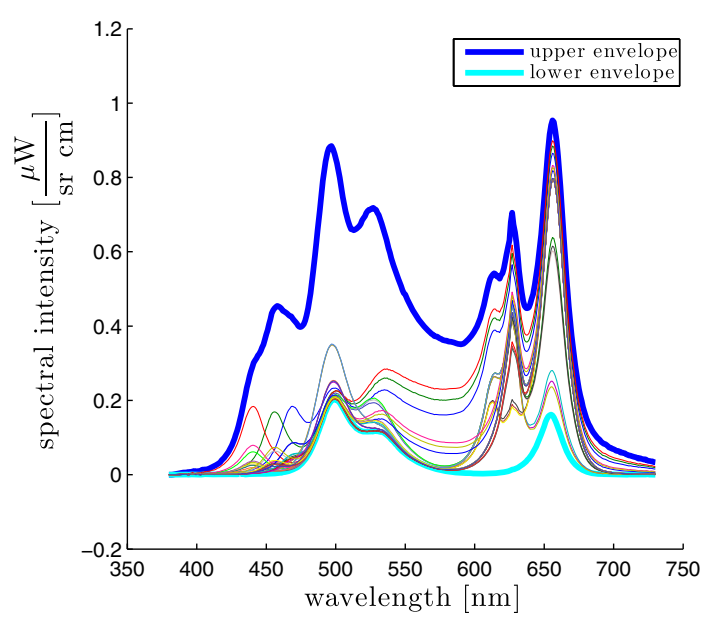

Fig. 16. Illuminant A normalized metamers calculated for one of the interpolation bands.

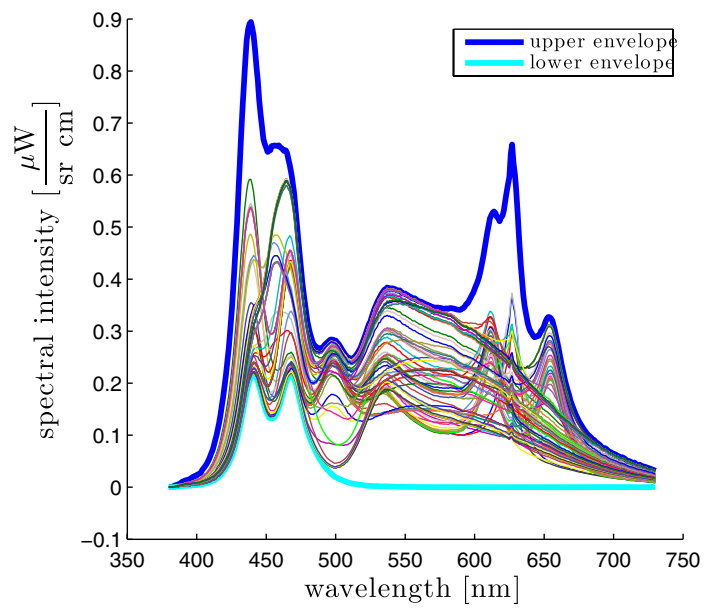

Fig. 17. Illuminant D65 normalized metamers calculated for one of the interpolation bands.

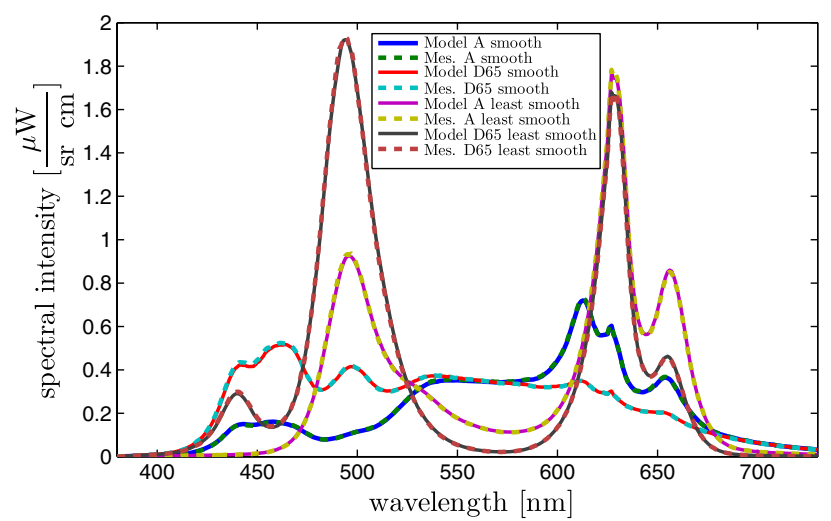

Fig. 18. Smoothest and least smooth illuminant A and D65 metamers at $246 \mathrm{~cd} / \mathrm{m}^{2}$ [according to the model (solid lines) and measured (dashed lines)].

from the complex algorithm and choosing the one candidate spectrum that meets the objective.

\section{CONCLUSIONS}

In this paper, we present methods for describing the metamer sets of illuminators comprising several light sources. Unlike the earlier work of Finlayson and Morovic, which aimed at inverting the color formation equation with respect to the reflectance spectrum, here we are using a similar approach and invert the color formation equation with respect to the illuminant spectrum. While the algorithms were developed and tested with a view to be used with a specific LED-based illuminator, they can be used for any light source that combines a number of channels and thus can be described by some linear model. The basic algorithm can be used for calculating the metamer sets of light sources that do not exhibit the intensity dependent channel spectra deformations. Many light sources fall into this category, e.g., the pulse-width modulation [28] based LED illuminators. As to the other light sources that exhibit the aforementioned spectra deformations (such as the one described in this work), a more complicated complex algorithm needs to be used. We have shown that a relatively coarse interpolation of the LED channels used in the complex algorithm provides an accurate description of the illuminator 
metamer set. Moreover, for this type of illuminator, we have defined the normalized metamer set describing all spectra physically realizable by the device that produce a given chromaticity response. Despite the coarse interpolation, the number of metamer sets that need to be calculated for the nonlinear LED illuminators may be significant, particularly if the number of such LED channels in the illuminator is higher than in the equipment considered in this paper. Further, in practice, although it appears that the number of metamer sets is large, most of them are empty (and so overall computational cost is reduced). Finally, we note that in practice, the choice of the LED channel intensity bands will depend on the number of LED channels, as well as their nonlinear properties, and will require some experimentation. We also presented an algorithm that selects the smoothest or the least smooth metamer from within the metamer sets obtained by either the basic or the complex metamer set algorithms.

\section{ACKNOWLEDGMENTS}

We would like to thank UK EPSRC for supporting this research (grants: EP/H022236/1 and EP/H022325/1).

\section{REFERENCES AND NOTES}

1. M. Mackiewicz, S. Crichton, S. Newsome, R. Gazerro, G. Finlayson, and A. Hurlbert, "Spectrally tunable led illuminator for vision research," in Proceedings of the 6th Colour in Graphics, Imaging and Vision (CGIV) (2012), Vol. 6, pp. 372-377.

2. G. Wyszecki and W. Styles, Color Science: Concepts and Methods, Quantitative Data and Formulae (Wiley, 1982).

3. G. Finlayson and P. Morovic, "Metamer sets," J. Opt. Soc. Am. A 22, 810-819, 2005.

4. P. Morovic, "Metamer sets," Ph.D. dissertation (School of Computing Sciences, University of East Anglia, 2002).

5. C. Lawson and R. Hanson, Solving Least-Squares Problems (Prentice-Hall, 1974).

6. M. S. Peercy, "Linear color representations for full speed spectral rendering," in Proceedings of the 20th Annual Conference on Computer Graphics and Interactive Techniques (SIGGRAPH '93) (ACM, 1993), pp. 191-198.

7. H. J. Trussell, Foundations of Digital Imaging (Cambridge University, 2008).

8. G. Golub and C. van Loan, Matrix Computations, 4th ed. (Johns Hopkins University, 2012).
9. J. B. Cohen, "Dependency of the spectral reflectance curves of the Munsell color chips," Psychon. Sci. 1, 369-370 (1967).

10. L. T. Maloney, "Evaluation of linear models of surface spectral reflectance with small numbers of parameters," J. Opt. Soc. Am. A 3, 1673-1683 (1986).

11. P. S. Parkkinen, J. Hallikainen, and T. Jaaskelainen, "Characteristic spectra of munsell colors,” J. Opt. Soc. Am. A 6, 318-322 (1989).

12. S. Westland, J. Shaw, and H. Owens, "Colour statistics of natural and man-made surfaces," Sensor Rev. 20, 50-55 (2000).

13. G. Wyszecki, "Evaluation of metameric colors," J. Opt. Soc. Am. 48, 451-454 (1958).

14. J. B. Cohen and W. E. Kappauf, "Metameric color stimuli, fundamental metamers, and Wyszecki's metameric blacks," Am. J. Psychol. 95, 537-564 (1982).

15. J. B. Cohen and W. E. Kappauf, "Color mixture and fundamental metamers: theory, algebra, geometry, application," Am. J. Psychol. 98, 171-259 (1985).

16. J. B. Fraleigh and R. A. Beauregard, Linear Algebra (AddisonWesley, 1990).

17. Finlayson and Morovic also describe an additional naturalness constraint, which is not relevant in this context. The interested reader can refer to [3].

18. B. Grünbaum, Convex Polytopes (Springer, 1967).

19. C. Barber, D. P. Dobkin, and H. T. Huhdanpaa, "The quickhull algorithm for convex hulls," ACM Trans. Mathematical Softw. 22, 469-483 (1996).

20. F. P. Preparata and M. I. Shamos, Computational Geometry An Introduction, 2nd ed. (Springer-Verlag, 1988).

21. Gamma Scientific, San Diego, USA. Available: http://www gamma-sci.com.

22. S. Muthu, F. J. Schuurmans, and M. D. Pashley, "Red, green, and blue LED based white light generation: issues and control," in Proceedings of the Institute of Electrical and Electronics Engineers Industry Applications Society Annual Meeting (IEEE IAS) (IEEE, 2002), Vol. 1, pp. 327-333.

23. C. Li and M. R. Luo, "The estimation of spectral reflectances using smoothness constraint condition," in Proceedings of the 9th Color and Imaging Conference (CIC) (2001), pp. 62-67.

24. P. E. Gill, W. Murray, and M. H. Wright, Practical Optimization (Academic, 1981).

25. R. Hunt and M. R. Pointer, Measuring Colour, 4th ed. (Wiley, 2011).

26. N. Ohta and G. Wyszecki, "Theoretical chromaticity mismatch limits of metamers viewed under different illuminants," J. Opt. Soc. Am. 65, 327-333 (1975).

27. A. Logvinenko, C. Godau, and B. Funt, "Metamer mismatch volumes," in Proceedings of the 6th Colour in Graphics, Imaging and Vision (CGIV), Amsterdam, 2012.

28. W. D. van Driel and X. J. Fan, Solid State Lighting Reliability (Springer, 2013). 